\title{
Tuning the Particle Sizes in Spherical Agglomeration
}

\section{Journal Article}

Author(s):

Orlewski, Pawel M.; Ahn, Byeongho; Mazzotti, Marco

Publication date:

2018-10-03

Permanent link:

https://doi.org/10.3929/ethz-b-000302128

Rights / license:

In Copyright - Non-Commercial Use Permitted

Originally published in:

Crystal Growth \& Design 18(10), https://doi.org/10.1021/acs.cgd.8b01134 


\title{
Tuning the Particle Sizes in Spherical Agglomeration
}

\author{
Pawel M. Orlewski, Byeongho Ahn, and Marco Mazzotti*(i) \\ Institute of Process Engineering, ETH Zurich, 8092 Zurich, Switzerland
}

Supporting Information

ABSTRACT: This paper presents an experimental technique for tuning the size of spherical agglomerates together with a simple model that provides qualitative information on the agglomerate size. Spherical agglomerates of benzoic acid of varying sizes using toluene as the bridging liquid were obtained with a very good reproducibility. The bridging liquid was injected into the crystal suspension via a capillary to control the initial size of the bridging liquid droplets. Experimental results obtained at different operating conditions show a clear correlation between the initial droplet size and the agglomerate size. Agglomerate size noticeably

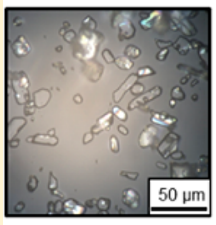

Primary Crystals

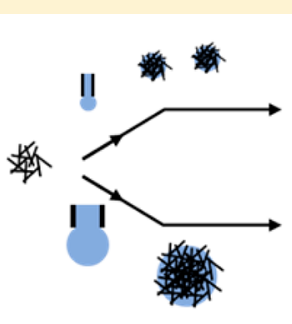

Binder Injection

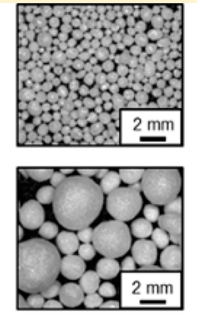

Spherical Agglomerates decreases with reducing the capillary size. Horizontal injection of the bridging liquid produces smaller agglomerates as compared to those produced with a vertical injection, while the size of agglomerates is insensitive to the different injection positions used in this study. Agglomerate size decreases along with the increasing stirring rate. The presented model adequately captures the behavior of the system and gives a qualitative agreement with the experimental results.

\section{INTRODUCTION}

Many chemical and pharmaceutical products are prepared as powders, often consisting of particles made of functional materials. The morphology of those particles plays a crucial role, as it can have an impact on the efficiency of downstream product handling (e.g., filtration and drying) and on the biopharmaceutical properties of particles (e.g., dissolution and bioavailability). ${ }^{1}$ Accordingly, a great deal of attention has been paid to modifying the crystal morphology during the crystallization phase by controlling the process parameters, e.g., supersaturation, cooling rate, stirring rate, or type of additives. However, sometimes undesired shapes or size distributions are inevitable, thus often forcing manufacturers to deal with micron-sized, needle-shaped, or platelet-shaped particles that exhibit poor flowability. This leads to a considerable decrease in the efficiency of the downstream processes such as filtration, blending, and compression (tableting).

Spherical crystallization has recently gained interest in the chemical and pharmaceutical industries to address the flowability-related problems by producing crystals in a spherical form with better micrometric properties such as flowability, packability, and compressibility compared to the needle- or platelet-shaped crystals. ${ }^{2}$ There are two types of spherical crystallization: quasi-emulsion solvent diffusion (QESD) and spherical agglomeration. In the case of the former, quasi-emulsion droplets of solvent in antisolvent are formed, within which crystallization occurs due to the counter diffusion of solvent out and antisolvent into the droplets. The latter on the other hand is induced by feeding a liquid binder (often called a bridging liquid), i.e., an immiscible fluid that preferentially wets the crystals, into the suspension, often after crystallization, but also possibly during crystallization. ${ }^{3}$ In such a process, the liquid binder is dispersed into a suspension of primary crystals in the form of droplets, which capture primary crystals by capillary action. ${ }^{4}$ Due to its higher flexibility and simplicity, preferential crystallization is considered more promising than QESD. ${ }^{3}$

Numerous studies have examined the effect of various operating parameters on the spherical agglomeration process: stirring rate, ${ }^{5,6}$ stirring time, ${ }^{4,6-8}$ initial size of primary crystals, $^{5,9}$ the amount, ${ }^{1,4,7,9}$ and the injection rate ${ }^{5,10}$ of the liquid binder. Among these parameters, often the amount of the liquid binder, together with its injection rate, has been shown to be the most critical parameter influencing the size of agglomerates. ${ }^{6,8,11}$ Surprisingly though, the influence of the initial binder dispersion, namely, the initial size of binder droplets, on the size of spherical agglomerates has not been studied so far.

The goal of this work is to understand the effect of the initial droplet size on the size of spherical agglomerates by exploiting the advantages offered by a microfluidic device, which enables the generation of a large quantity of binder droplets, whose size can be carefully tuned. It also allows decoupling the features of the initial binder dispersion from other critical operating parameters, thus enabling a better understanding of the role of the liquid binder in the spherical agglomeration process.

\section{TUNING THE SIZE OF DROPLETS: THEORY}

Advances in microfluidics have facilitated droplet generation in a controlled manner, as summarized in a recent review paper. ${ }^{12}$ Among the numerous techniques for droplet generation, we

Received: July 27, 2018

Revised: September 3, 2018

Published: September 5, 2018 
have employed the simplest method, i.e., injecting the dispersed phase into the continuous (immiscible) liquid phase via a cylindrical microcapillary. In this method, under certain flow conditions, the droplet forms at the capillary tip and further grows until it reaches a critical size, after which the droplet starts to detach, thus eventually leaving the capillary (see a high-speed camera video of the whole process in the Supporting Information). The size of the detached droplet can vary depending on the capillary size, the flow field around the capillary tip, and the properties of the fluids involved and of the capillary. ${ }^{13}$ Since the droplet size is at the heart of this work, the following section presents a simple model that has been used to estimate such a droplet size.

2.1. Droplet Size Estimation. There are four main forces acting on a droplet forming at the tip of a capillary, namely, the buoyancy force $F_{\mathrm{B}}$, the kinetic force $F_{\mathrm{K}}$, the drag force $F_{\mathrm{D}}$, and the interfacial tension force $F_{\mathrm{I}}$. The aforementioned forces can be classified into two groups: lifting forces, that act to separate the droplet from the tip, and restraining forces, that act to keep it attached to the tip. ${ }^{14}$ If the flow directions of the continuous and dispersed phase are assumed to be parallel (Figure 1), then

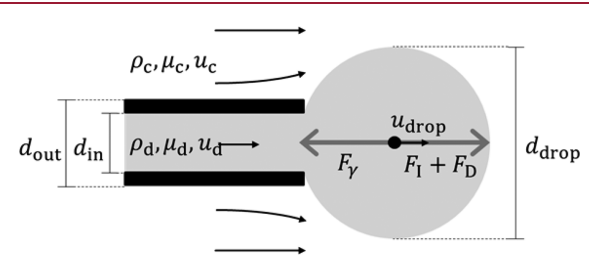

Figure 1. Schematic drawing of the droplet formation at the capillary tip in the dripping regime. The dispersed phase is injected through a capillary into a co-flowing immiscible outer fluid. Three major forces acting on the droplets are displayed with solid arrows: interfacial tension force $F_{\gamma}$, inertial force $F_{\mathrm{I}}$, and drag force $F_{\mathrm{D}}$.

the only force keeping the droplet attached to the nozzle is the interfacial tension force, whereas the remaining three forces act as lifting forces. As long as the restraining forces are larger than the lifting forces, the droplet will stay attached to the tip and continue growing. As soon as the lifting forces exceed the restraining forces, the droplet will start clearing the tip of the capillary. ${ }^{14}$ The size of droplets at the breaking point corresponds to the size of a droplet, for which the restraining forces are balanced by the lifting forces. Below, the definitions of the four forces acting on the droplet are provided.

The interfacial tension force $F_{\gamma}$, that keeps the droplet at the capillary tip, is given by

$$
F_{\gamma}=\frac{\pi \gamma\left(d_{\text {in }}+d_{\text {out }}\right)}{2}
$$

where $\gamma$ is the interfacial tension between the two fluids, and $d_{\text {in }}$ and $d_{\text {out }}$ are the inner and outer diameter of the capillary, respectively. Here, the perimeter of the triple interface has been defined as $\pi\left(d_{\text {in }}+d_{\text {out }}\right) / 2$ instead of the usual $\pi d_{\text {in }}$, because the actual perimeter of the triple-phase contact line among the two fluids and the capillary tip is somewhere in between $\pi d_{\text {in }}$ and $\pi d_{\text {out }}$, and well approximated by their arithmetic average. $^{15}$

The inertial force $F_{\mathrm{I}}$, that is generated due to the momentum of the dispersed phase inside the capillary and that pushes the droplet in the streamwise direction, is defined as

$$
F_{\mathrm{I}}=\rho_{\mathrm{d}} q_{\mathrm{d}} u_{\mathrm{d}}
$$

where $\rho_{\mathrm{d}}$ is the density of the dispersed phase, $u_{\mathrm{d}}$ is its average velocity inside the capillary, and $q_{\mathrm{d}}$ is its volumetric flow rate.

The drag force, which also acts to detach the droplet from the capillary, is given by

$$
F_{\mathrm{D}}=\frac{C_{\mathrm{D}} \rho_{\mathrm{c}} u_{\mathrm{rel}}^{2} A_{\mathrm{ref}}}{2}
$$

where $C_{D}$ is the drag coefficient (dimensionless), $\rho_{c}$ is the density of the continuous phase, $u_{\text {rel }}$ is the relative velocity of the continuous phase with respect to the droplet, and $A_{\text {ref }}$ is the reference area. The drag coefficient $C_{D}$ can be estimated as a function of the Reynolds number $R e$ as follows: ${ }^{16}$

$$
C_{\mathrm{D}}=0.292\left(1+\frac{9.06}{\sqrt{R e}}\right)^{2}
$$

Here, the Reynolds number $R e$ is defined as ${ }^{17}$

$$
R e=\frac{\rho_{\mathrm{c}} u_{\text {rel }}\left(d_{\mathrm{drop}}-d_{\text {out }}\right)}{\mu_{\mathrm{c}}}
$$

where $d_{\text {drop }}$ is the diameter of the droplet and $\mu_{\mathrm{c}}$ is the dynamic viscosity of the continuous phase. The term $d_{\text {drop }}-d_{\text {out }}$ reflects the fact that the capillary shields the inner side of the droplet from the continuous phase, as shown in Figure 1. The relative velocity of the continuous phase with respect to the droplet $u_{\text {rel }}$ is $u_{\mathrm{c}}-u_{\text {drop }}$, where $u_{\mathrm{c}}$ is the average velocity of the continuous phase, $u_{\text {drop }}$ is the velocity of the droplet in the streamwise direction, and it is given by $q_{\mathrm{d}} /\left(\pi d_{\mathrm{drop}}^{2}\right)$. The reference area $A_{\text {ref }}$ is typically defined as the area of an object perpendicular to the flow direction. In this work, it is defined as ${ }^{17}$

$$
A_{\text {ref }}=\frac{\pi\left(d_{\text {drop }}^{2}-d_{\text {out }}^{2}\right)}{4}
$$

which accounts for the fact that the droplet is partially shielded from the continuous phase flow by the capillary itself, as illustrated in Figure 1.

The buoyancy force $F_{\mathrm{B}}$, that is generated due to the density difference between the two phases, is defined as

$$
F_{\mathrm{B}}=V_{\mathrm{d}} g \Delta \rho
$$

where $V_{\mathrm{d}}$ is the volume of the droplet, $g$ is the acceleration of gravity, and $\Delta \rho$ is the difference between the densities of the two phases. Under the investigated conditions, the buoyancy force is negligible in comparison with the other three forces and therefore has been neglected; this is often the case in liquid-liquid drop formation. ${ }^{13,17-19}$

By balancing the three horizontal forces, the size of droplets at the breaking point can be calculated:

$$
F_{\gamma}=F_{\mathrm{I}}+F_{\mathrm{D}}\left(d_{\text {drop }}, \mathrm{u}_{\mathrm{c}}\right)
$$

where only the drag force $F_{D}$ increases with the droplet size $d_{\text {drop, }}$, whereas the interfacial tension force $F_{\gamma}$ and the inertial force $F_{\mathrm{I}}$ are independent of $d_{\text {drop }}$. Here, the average velocity of the continuous phase $u_{c}$ used in the drop size estimation is obtained from computational fluid dynamics (CFD) simulations, as explained in Section 2.2.

There are two main limitations to this model. First, the flow directions of the continuous and dispersed phase are assumed to be parallel (referred in this work as the co-flow configuration). In the case of cross-flow, the droplet breaks off from the external surface of the capillary rather than from 
the tip, thus providing a larger contact area between the droplet and the capillary, and a larger shielding effect from the continuous phase as compared to the co-flow case (Figure 3). This increases the effect of the surface tension force and decreases the drag force, thus resulting in a larger droplet size at break-off. Unfortunately, the actual break-off point is not known, and therefore the droplet size cannot be precisely estimated. Since developing a new model for droplet formation lays beyond the scope of this paper, we have decided to use the simple model.

Second, the droplets form in the "dripping regime" where the dispersed phase drips from the capillary tip in the form of droplets, thus being not applicable to the "jetting regime" drop formation where the dispersed phase forms a liquid jet and breaks up into droplets due to Rayleigh instabilities. ${ }^{20}$

To check the validity of the second assumption, a theoretical approach to characterize the flow regime of droplet formation has been employed by comparing the kinetic energy per unit length with the interfacial tension energy per unit length. ${ }^{17}$ When the kinetic energy term is smaller than the interfacial energy term, droplets form under the dripping regime. The kinetic energy of pipe flow per unit length is $\rho_{\mathrm{d}} u_{\mathrm{d}}^{2} d_{\mathrm{in}}^{2} / 8$, where $\rho_{\mathrm{d}}$ is the density of the dispersed phase, $u_{\mathrm{d}}$ is the average velocity of the dispersed phase inside the capillary, and $d_{\text {in }}$ is the inner diameter of the capillary. The interfacial tension energy per unit length is $\pi \gamma\left(d_{\text {in }}+d_{\text {out }}\right) / 2$. In this work, the interfacial tension energy term is always larger than the kinetic energy term, thus implying that the dripping regime governs the drop formation. It is, however, important to note that this approach does not consider the energy transferred from the coflowing continuous phase to the droplet, which can influence the drop formation mechanism. ${ }^{13}$

2.2. CFD Model. In order to get a better understanding of the flow conditions in the reactor and to obtain the local fluid velocities needed for the estimation of the size of the generated droplets, a CFD model of the crystallizer has been developed using the commercial CFD software Fluent 17.2. As significant vortex formation due to the intensity of mixing has been observed during the experiments, a two-phase model was used to capture this effect. To solve the Navier-Stokes equations, a standard Reynolds stress model (RSM) with linear pressurestrain has been used together with the volume of fluid (VOF) multiphase model where the liquid phase has been modeled as pure water and the gas phase as air. The simulations were run at steady state using the in-built multi reference frame (MRF) model for the description of the motion of the stirrer. To reduce the computational burden, periodic boundary conditions have been used with only a quarter of the reactor (having a volume of $500 \mathrm{~mL}$, see section 3.2) being sufficient to describe the whole system. The grid has been refined until a mesh-independent solution was obtained with the final grid consisting of 237000 cells.

\section{EXPERIMENTAL SECTION}

Spherical agglomeration of benzoic acid crystals using toluene as a bridging liquid has been investigated in this work, whose main goal is to study the effect of the initial droplet distribution of the bridging liquid on the final particle size distribution (PSD) of the spherical agglomerates. Therefore, the experiments have been designed (1) to study the effect of the initial droplet size distribution on the final size distribution of the spherical agglomerates (series $E_{1}$ ), (2) to establish the effect of feeding position on the final size of agglomerates (series $E_{2}$ ), and (3) to determine which effect dominates in determining the final size of agglomerates-either the agglomeration kinetics or the initial dispersion of the bridging liquid (series $E_{3}$ ). Each experiment consisted of two stages: first the unseeded generation of primary crystals (stage I), carried out under the same conditions for all the experiments, followed by the agglomeration of the primary crystals into spheres (stage II), performed using different operating conditions to explore the effects described above.

3.1. Materials. In this work, benzoic acid (99.5\% purity, SigmaAldrich, Switzerland) has been used as solute, ethanol (absolute, Fischer Chemicals, Switzerland) as the solvent, ultrapure deionized water $(18.2 \mathrm{M} \Omega \mathrm{cm}$, Millipore, Switzerland) as the antisolvent, and toluene (HPLC grade, Fischer Chemicals, Switzerland) as the bridging liquid. The physical properties of the liquids at room temperature are summarized in Table 1.

Table 1. Physical Properties of Liquids Used in This Work at Room Temperature ${ }^{a}$

\begin{tabular}{llccc}
\multicolumn{1}{c}{ phase } & \multicolumn{1}{c}{ composition } & $\begin{array}{c}\rho \\
{\left[\mathrm{kg} / \mathrm{m}^{3}\right]}\end{array}$ & $\begin{array}{c}\mu \\
{[\mathrm{mPa} \mathrm{s}]}\end{array}$ & $\gamma[\mathrm{mN} / \mathrm{m}]$ \\
continuous & water/ethanol $(90 / 10)$ & $963^{b}$ & $1.86^{b}$ & $20^{c}$ \\
dispersed & toluene & 865 & 0.56 &
\end{tabular}

${ }^{a}$ Mixture proportions are given in w/w. ${ }^{b}$ Reference $21 .{ }^{c}$ Reference 22.

3.2. Experimental Setup. All experiments have been carried out as a semibatch process using a $500 \mathrm{~mL}$ curved-bottom cylindrical vessel with a diameter $D=10 \mathrm{~cm}$ (GlasKeller, Germany) (see Figure 2). The temperature inside the reactor was controlled using a Huber

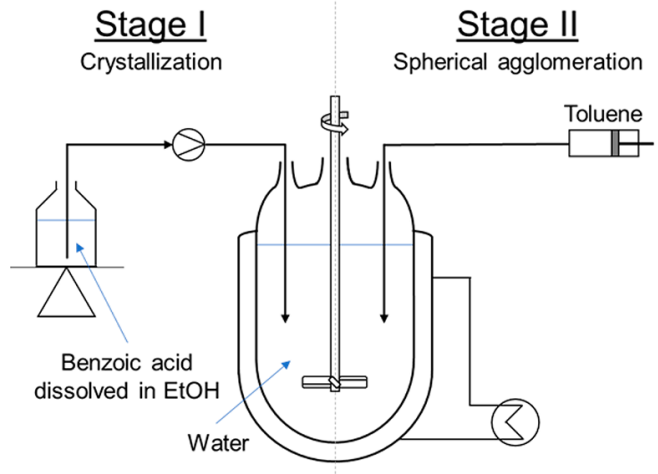

Figure 2. Overview of the experimental setup.

Ministat CC3 thermostat (Huber, Germany). The solution was agitated using an overhead, four-blade glass impeller with a diameter of $75 \mathrm{~mm}$ and $40^{\circ}$ inclined blades. The impeller was powered by a Eurostar Digital stirrer (IKA, Germany) and positioned $25 \mathrm{~mm}$ above the reactor bottom with the shaft turning counterclockwise, thus resulting, in this case, in an upward conveying flow direction. The lid of the reactor was made of glass and had ports for a thermocouple, a liquid inlet used for antisolvent crystallization, and a liquid inlet used for spherical agglomeration.

Stage I of the process-unseeded antisolvent crystallization-was performed by feeding an undersaturated solution of benzoic acid in the solvent (ethanol) to the reactor filled with the antisolvent (water). The stock solution was placed on a balance (Mettler Toledo, Switzerland) and fed to the reactor using a LabAlliance Series II HPLC pump (Scientific Systems, USA), thus enabling a precise dosage of the solution through the process. The feeding tube was located $1 \mathrm{~cm}$ above the stirrer and $2 \mathrm{~cm}$ away from the shaft.

Stage II of the process-spherical agglomeration-was carried out by feeding the bridging liquid (toluene) to the reactor containing crystals suspended in a saturated solution. The bridging liquid was introduced using a PHD4400 syringe pump (Harvard Apparatus, USA). Due to the high pressure drop in the system, a $20 \mathrm{~mL}$ stainless steel syringe (Harvard Apparatus, USA) has been used to ensure 
precise dosage for all the process conditions investigated in this work. As feeding tubes, fused silica capillaries (BGB Analytik AG, Switzerland) with four different sizes were selected with different inner and outer diameters, $d_{\text {din }}$ and $d_{\text {out }}$ respectively (see Table 2). To

Table 2. Inner and Outer Diameter of Capillaries Used for Feeding Bridging Liquid

$\begin{array}{ccc}\text { capillary } & d_{\text {in }}[\mu \mathrm{m}] & d_{\text {out }}[\mu \mathrm{m}] \\ G_{1} & 100 & 164 \\ G_{2} & 250 & 360 \\ G_{3} & 320 & 735 \\ G_{4} & 500 & 794\end{array}$

protect them from mechanical damage and to allow for a precise positioning of the inlet tip in the reactor, the feeding tubes have been guided within the reactor through a metal housing (see Figure 3).
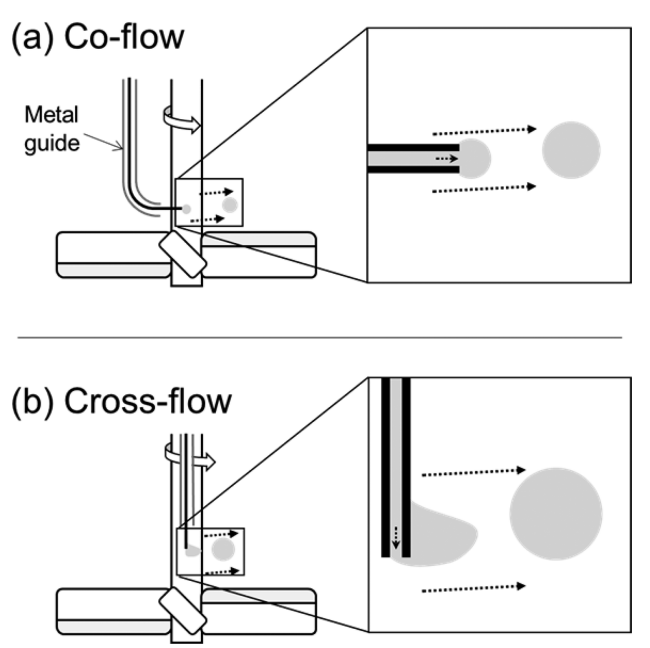

Figure 3. Schematic drawings of droplet break-off in a (a) co-flow and (b) cross-flow configuration. The dispersed phase (gray) is pumped into a reactor through a fused-silica capillary (black), which is covered by a metal guide. The droplet break-off location is the capillary tip in the co-flow configuration, whereas it is the external surface of the capillary in the cross-flow configuration.

Two different flow configurations have been used for feeding the bridging liquid. In the first case, the inlet was located in a horizontal position and parallel to the direction of the flow (see Figure 3a), whereas in the second case, the inlet was positioned vertically (see Figure $3 \mathrm{~b}$ ), thus resulting in co-flow and cross-flow conditions at the tip, respectively.

3.3. Experimental Procedure. 3.3.1. Stage I: Preparation of Primary Crystals. The feed solution has been prepared by dissolving benzoic acid in ethanol to obtain the chosen concentration, $c_{0, \mathrm{BA}}=0.5$ $\mathrm{g}_{\text {solute }} / \mathrm{g}_{\text {solvent }}$. The experiments were run at a constant temperature, $T$ $=25{ }^{\circ} \mathrm{C}$. First, water $(409.09 \mathrm{~g})$ was loaded into the reactor and thermostated under agitation until reaching a constant temperature. In the meantime, the HPLC pump was primed with the benzoic acid solution. Next, a stirring rate of $250 \mathrm{rpm}$ was set, and the benzoic acid solution $(61.36 \mathrm{~g})$ was fed at a constant flow rate, $Q_{\text {feed }}=10 \mathrm{~mL} / \mathrm{min}$. Afterward, the slurry was left to age for $30 \mathrm{~min}$.

The reproducibility of the primary crystals properties has been checked for three independent repetitions using laser diffraction (Sympatec, Germany). As shown in Figure 4 reproducibility in terms of particle size distribution (PSD) of the primary crystals was satisfactory (Figure 4).

3.3.2. Stage II: Spherical Agglomeration. As in the previous stage, the experiments were run at a constant temperature, $T=25^{\circ} \mathrm{C}$. Toluene was placed in a $20 \mathrm{~mL}$ stainless steel syringe which was later

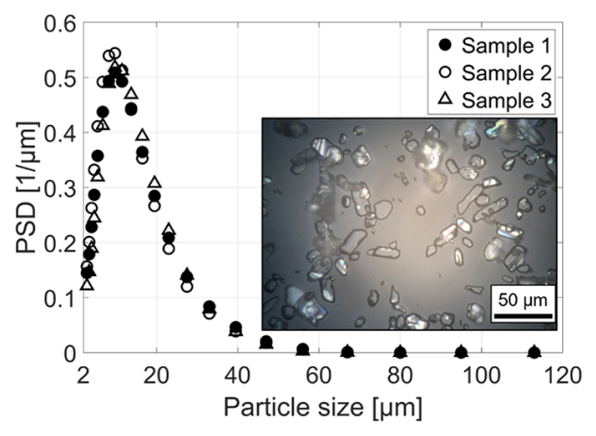

Figure 4. PSD of primary crystals measured by laser diffraction. Each sample was produced from a different batch. On the microscopic image of the primary crystals, small needles of benzoic acid can be observed.

installed in the pump. The feeding pipe was prepared by carefully cutting the silica capillary. Every time a new capillary was prepared, microscopic pictures of the outlet tip were taken and evaluated, to make sure that the cut was clean and that the tip was not damaged. The feeding pipe was primed and, depending on the desired flow configuration at the tip, guided through one of the two metal guides positioned in the crystallizer. Afterward, the bridging liquid inlet was installed in the reactor, making sure that the positioning of the tip was precise, i.e., at a distance $r$ from the stirrer shaft and at the position $z$ in the vertical direction in one of the four locations investigated. Next, the desired stirring rate was set, and toluene $(12.80 \mathrm{~mL})$ was fed at a constant flow rate, $Q_{\text {feed }}=0.5 \mathrm{~mL} / \mathrm{min}$, thus resulting in a ratio of the volume of the bridging liquid to the mass of crystals (BSR) of 0.7 $\mathrm{mL} / \mathrm{g}$. Afterward, the suspension was left to age for 55 min under agitation. Finally, the crystalline product was filtered using vacuum filtration and left to dry for $12 \mathrm{~h}$ under ambient conditions. The final product size distribution was obtained using microscopic images and image analysis.

\section{RESULTS AND DISCUSSION}

As shown in Figure 5, spherical agglomerates of benzoic acid of varying sizes have been successfully obtained. As mentioned above (see Section 1), the values of the feeding rate of the bridging liquid, of the ratio of the volume of bridging liquid to the mass of crystals, and of the resulting feeding time of the bridging liquid are crucial for obtaining spherical agglomerates. Such values were chosen so round, compact, and strong
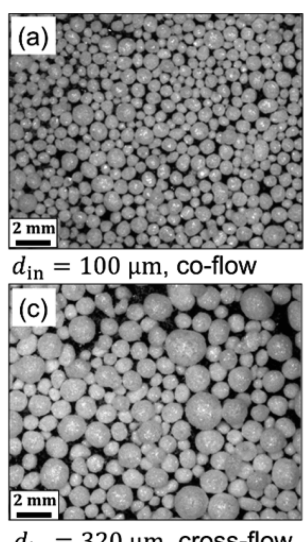

$d_{\text {in }}=320 \mu \mathrm{m}$, cross-flow

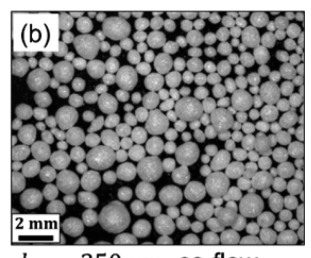

$d_{\text {in }}=250 \mu \mathrm{m}$, co-flow

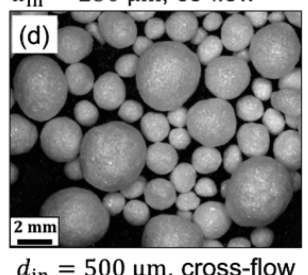

Figure 5. Microscopic images of spherical agglomerates produced with different $d_{\text {in }}$ and flow configurations. In those experiments, the feeding position $(z)$ and stirring rate was set to $2.64 \mathrm{~cm}$ and $450 \mathrm{rpm}$, respectively. The corresponding volume-averaged sizes have been reported in Figure $7 \mathrm{a}-\mathrm{d}$. 
Table 3. Details of the Experimental Conditions for the Three Types of Experiments Performed

\begin{tabular}{|c|c|c|c|c|c|}
\hline \multirow[b]{2}{*}{ group } & \multirow[b]{2}{*}{ capillary } & \multicolumn{2}{|c|}{ feed position } & \multirow[b]{2}{*}{ stirring rate $\left[\mathrm{min}^{-1}\right]$} & \multirow[b]{2}{*}{ flow configuration } \\
\hline & & $r[\mathrm{~cm}]$ & $z[\mathrm{~cm}]$ & & \\
\hline$E_{1}$ & $G_{1}, G_{2}, G_{3}, G_{4}$ & 3.8 & 2.64 & 450 & co-flow, cross-flow \\
\hline$E_{2}$ & $G_{3}$ & $\begin{array}{l}3.8 \\
2.8\end{array}$ & $\begin{array}{c}-1.34,1.14,2.64 \\
-2.64\end{array}$ & 450 & co-flow \\
\hline$E_{3}$ & $G_{3}$ & 2.8 & -2.64 & $250,350,450$ & co-flow \\
\hline
\end{tabular}

spherical agglomerates could be produced and were kept constant for all the experiments (see Section 3.3.2). To study the effect of the initial droplet size on the final size of agglomerates, spherical agglomerates were produced at different operating conditions with four other process parameters being varied: inner diameter of the feeding capillary $d_{\text {in }}(100$, 250, 320, $500 \mu \mathrm{m}$ ), flow configuration (co-flow, cross-flow), feed position $\mathrm{z}(-2.64,-1.34,1.14,2.64 \mathrm{~cm})$, and stirring rate $(250,350,450 \mathrm{rpm})$ (see Table 3 ). Since the droplets were generated in the dripping regime (see Section 2.1), the size of the generated droplets could not be smaller than that of the capillary and was always larger than the average size of the primary crystals. The volume-averaged size $d_{43}$ of the resulting agglomerates ranged from 800 to $2200 \mu \mathrm{m}$ with a very good reproducibility in terms of both the average size and the particle size distribution (PSD) of the final agglomerates.

4.1. Effect of Bridging Liquid Droplet Size on Agglomerate Size (Series $E_{1}$ ). The main goal of this work is to understand the role played by the bridging liquid droplet size $\left(d_{\text {drop }}\right)$ in spherical agglomeration. As explained in Section $2.1, d_{\text {drop }}$ is proportional to the inner diameter of the capillary $d_{\text {in }}$ and is expected to decrease with decreasing $d_{\text {in }}$. Moreover, $d_{\text {drop }}$ is also expected to be smaller when the flow directions of the continuous phase and the dispersed phase are "parallel" (co-flow configuration) than when they are "perpendicular" (cross-flow configuration), as illustrated in Figure 3. To demonstrate a correlation between $d_{\text {drop }}$ and the final size of spherical agglomerates, a series of experiments $\left(E_{1}\right)$ were performed in which only $d_{\text {drop }}$ was varied (by changing $d_{\text {in }}$ and/ or the flow configuration) while keeping the other operating conditions (stirring rate, stirring time, initial size, and amount of primary crystals, amount and injection rate of the liquid binder, feed position $z$ ) constant (see Table 3). Therefore, the difference in size of agglomerates between different experiments can only be attributed to the change in the initial droplet size.

In Figure 6, the PSDs of the final agglomerates obtained with different feeding capillaries and flow configurations are plotted. The experimental results demonstrate that in general the size of spherical agglomerates increases with $d_{\text {in }}$ for both flow configurations. In other words, the variation of droplet size by changing $d_{\text {in }}$ allows tuning of the agglomerate size. With an increase in $d_{\text {in }}$, both the average and the variance of the PSD increase, as shown in Figure 6. This is mainly because a capillary with larger $d_{\text {in }}$ produces larger bridging liquid droplets (larger $\left.d_{\text {drop }}\right)$. On the one hand, larger droplets collect a larger amount of primary crystals, hence yielding larger agglomerates. On the other hand, some large droplets can break up into small droplets, thus resulting in droplets with a broad size distribution, which consequently broadens the size distribution of the final agglomerates.

In Figure 7, the dependence of the volume-averaged size ( $d_{43}=m_{4} / m_{3}$, where $m_{4}$ and $m_{3}$ are the fourth and third
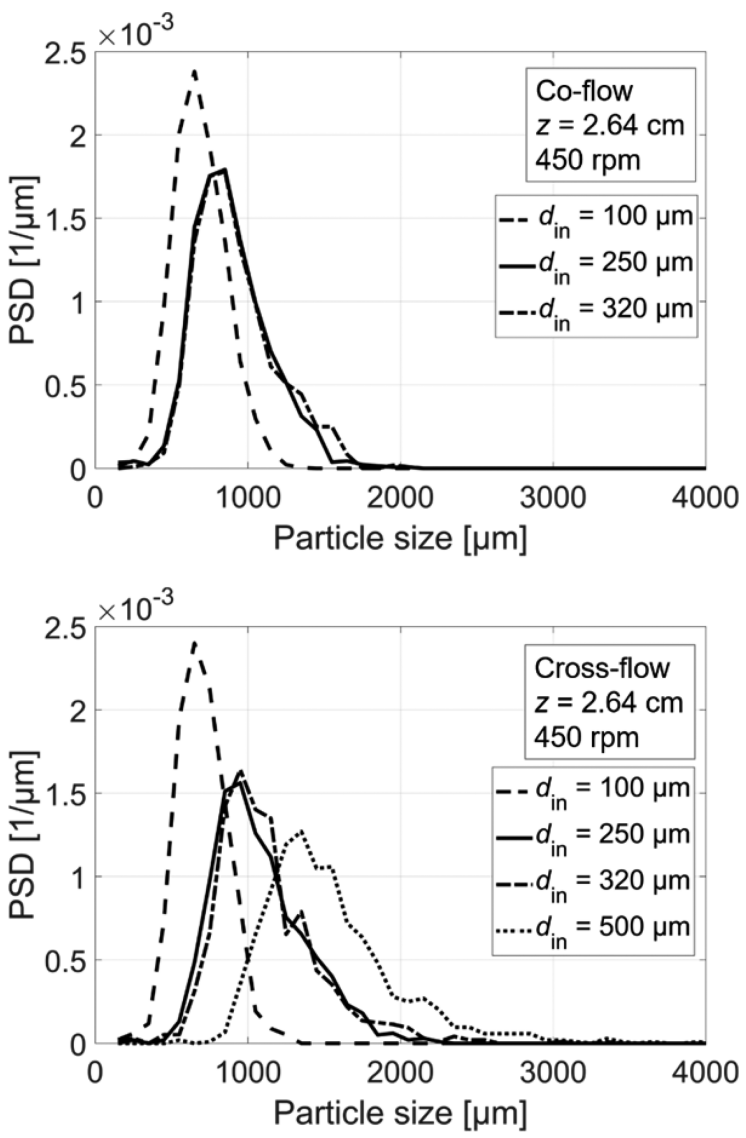

Figure 6. Effect of $d_{\text {in }}$ on the PSD of spherical agglomerates in a coflow configuration (top) and cross-flow configuration (bottom) for experiments $E_{1}: z=2.64 \mathrm{~cm}$, stirring rate $=450 \mathrm{rpm}$. All experiments were performed twice, yielding very similar PSDs. For the sake of clarity, only one of the two experimental results is reported for each experimental condition.

moments of the relevant distribution plotted in Figure 6) of spherical agglomerates on $d_{\text {in }}$ is illustrated.

As it can readily be seen, $d_{43}$ of spherical agglomerates varies strongly with the capillary diameters $d_{\text {in }}$, and more weakly with the flow configuration, clearly proving that the agglomerate size can be varied with an adjustment in $d_{\text {drop }}$ (by changing $d_{\text {in }}$ or the flow configuration). In the case of a cross-flow configuration, $d_{43}$ increases from 800 to $2200 \mu \mathrm{m}$ by simply changing $d_{\text {in }}$ from 100 to $500 \mu \mathrm{m}$. Moreover, for $d_{\text {in }}=320 \mu \mathrm{m}$, $d_{43}$ increases from 1200 to $1550 \mu \mathrm{m}$ by switching the flow configuration from co-flow to cross-flow.

4.2. Comparison with the Droplet Generation Model. To complement our experimental study, a mathematical model has been used to estimate the size of bridging liquid droplet by solving eq 8 under the assumption of a co-flow configuration (see Section 2.1). As mentioned above (see Section 2.2), the velocities of the continuous phase in the reactor required to 


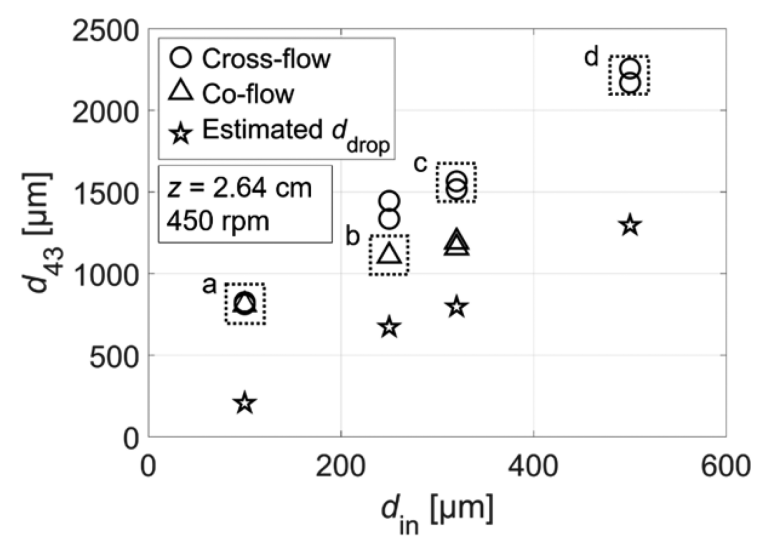

Figure 7. Effect of $d_{\text {in }}$ on the volume-averaged size $\left(d_{43}\right)$ of spherical agglomerates (circles and triangles) and the corresponding $d_{\text {drop }}$ estimated from eq 8 (stars) for experiments $E_{1}: z=2.64 \mathrm{~cm}$, stirring rate $=450 \mathrm{rpm}$. Microscopic images of the four different sets of agglomerates indicated by $\mathrm{a}-\mathrm{d}$ have been reported in Figure 5 .

solve the force balance have been obtained from the CFD simulations.

Contour plots of the velocity magnitude in the reactor for the three investigated stirring rates, namely, 250, 350, and 450 rpm, are shown in Figure 8. Since the radial and axial components of the velocity field are orders of magnitude smaller than the tangential component, the absolute value of the tangential component is essentially equal to the velocity magnitude, which is then used for the calculations. The average velocities of the continuous phase at a distance $r$ from the stirrer shaft and position $\mathrm{z}$ in the vertical direction, $\bar{u}_{\mathrm{c}}(r, z)$, required for the estimation of the size of the generated droplets, were calculated using the following expression:

$$
\bar{u}_{\mathrm{c}}(r, \mathrm{z})=\int_{0}^{\infty} u_{\mathrm{c}}(r, \mathrm{z}) f\left(u_{\mathrm{c}}(r, \mathrm{z})\right) \mathrm{d} u
$$

where $f\left(u_{c}(r, z)\right)$ is the position-dependent probability distribution function of the fluid velocity in the reactor. The statistical properties of the distributions of $u_{\mathrm{c}}(r, z)$ for the $r$ and $\mathrm{z}$ investigated in this work are also shown in Figure 9. As expected, the average velocity of the fluid increases with an increasing stirring rate (Figure 9, 1a-1c). For the four investigated feeding locations at the stirring rate of $450 \mathrm{rpm}$, the average velocity as well as the width of the distribution

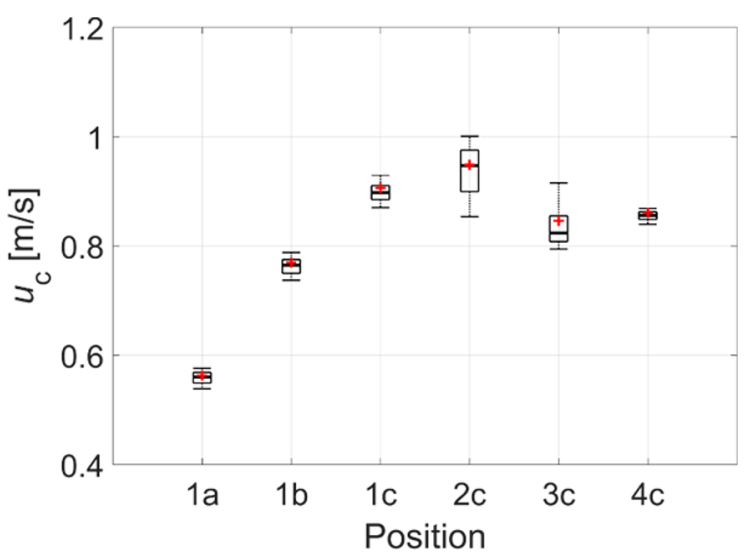

Figure 9. Box-plot of the distribution of $u_{\mathrm{c}}$ at various investigated feeding positions in the reactor. The band inside the box represents the median with the box representing first and third quantile of the distribution. Lower and upper whiskers correspond to the 9th and 91st percentiles of the distribution, respectively. The mean of the distribution $\left(\bar{u}_{c}\right)$ has been indicated with a red cross.

changes depending on the feeding position with more uniform velocity flow-fields away from the impeller and slightly higher average velocities below the impeller (Figure 9, 1c-4c). In Table 4, the obtained average velocities as well as the corresponding estimated initial droplet sizes obtained when using the $\mathrm{G}_{3}$ capillary are reported.

Table 4. Average Velocities of the Continuous Phase at the Investigated Locations in the Reactor and Stirring Rates with the Corresponding Estimated Initial Droplet Size for the $G_{3}$ Capillary (Experiments $E_{2}$ and $E_{3}$ )

\begin{tabular}{|c|c|c|c|c|}
\hline \multicolumn{2}{|c|}{ feed position } & \multirow[b]{2}{*}{ stirring rate $\left[\mathrm{min}^{-1}\right]$} & \multirow[b]{2}{*}{$\bar{u}_{\mathrm{c}}[\mathrm{m} / \mathrm{s}]$} & \multirow[b]{2}{*}{$d_{\mathrm{drop}}[\mu \mathrm{m}]$} \\
\hline$r[\mathrm{~cm}]$ & $z[\mathrm{~cm}]$ & & & \\
\hline 3.8 & 2.64 & 450 & 0.86 & 788 \\
\hline 3.8 & 1.14 & 450 & 0.85 & 792 \\
\hline 3.8 & -1.34 & 450 & 0.95 & 755 \\
\hline 2.8 & -2.64 & 450 & 0.91 & 769 \\
\hline 2.8 & -2.64 & 350 & 0.77 & 830 \\
\hline 2.8 & -2.64 & 250 & 0.56 & 977 \\
\hline
\end{tabular}

the average velocity as well as the width of the distribution

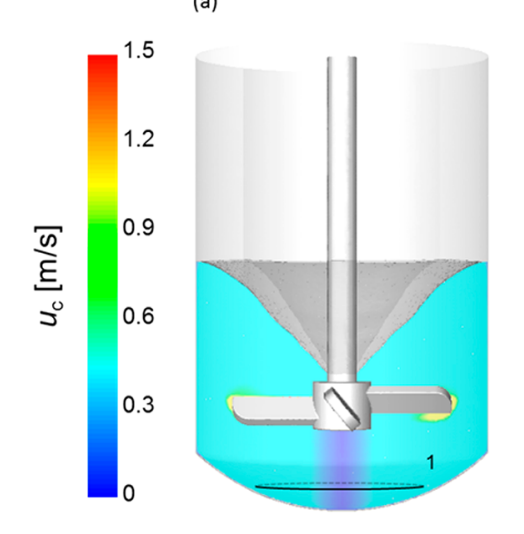

(b)

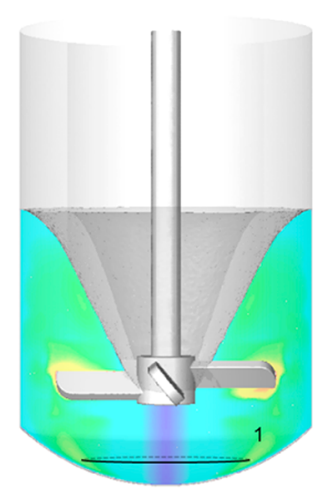

(c)

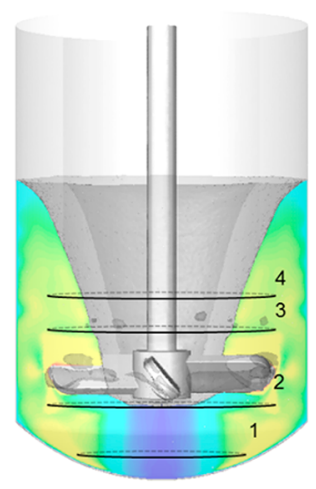

Figure 8. Contour plots of the velocity magnitude of the liquid phase in the reactor in the $y-z$ plane for the three investigated stirring rates: (a) 250 $\mathrm{rpm}$, (b) $350 \mathrm{rpm}$, and (c) $450 \mathrm{rpm}$. The gray surface corresponds to the gas-liquid interface. Each black circle corresponds to one of the feeding positions used in the experiments. 
In Figure 7, the droplet sizes estimated with different sizes of capillaries are plotted using star markers. For all the cases investigated in this work, the estimated $d_{\text {drop }}$ is always considerably smaller than the corresponding average size of agglomerates $d_{43}$. This observation is not surprising, but in agreement with the mechanism of spherical agglomeration proposed in the literature (see Figure 10 for an illustration of

1) Wetting

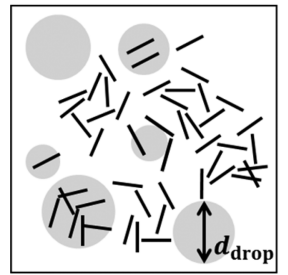

2) Compaction

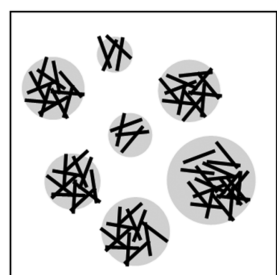

3) Growth

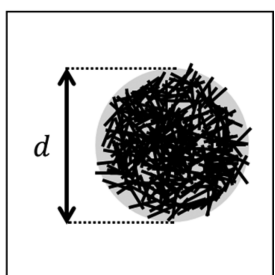

Figure 10. Illustration of the three steps governing the spherical agglomeration process: (1) formation of the agglomerate nuclei, (2) compaction of the agglomerate nuclei, (3) growth of the agglomerates due to coalescence. Black segments represent the primary crystals, whereas the gray circles represent the bridging liquid droplets.

it). It has been found that the process of spherical agglomeration can be described as a three-step process. ${ }^{6,23,24}$ In the first step, often referred to as the "wetting-step", droplets of the bridging liquid capture primary crystals and form the socalled agglomerate nuclei. This is followed by the "compactionstep" where, due to the collisions with other particles, agglomerate nuclei are compacted. The last step is the "growth-step", in which agglomerates grow by a coalescence process, i.e., by colliding with each other thus forming larger agglomerates, whose final size may be much larger than the initial size of the dispersed bridging liquid droplets.

Furthermore, by comparing the estimated $d_{\text {drop }}$ with the average size of agglomerates $d_{43}$, a clear correlation can be observed. This further confirms the previous assumption that an increase in the inner diameter of the capillary leads to an increase in the droplet size, which consequently results in larger agglomerates. Despite using a very simplified model of droplet formation, the estimated droplet size captures very well the key trends in the behavior of the system under the investigated conditions (constant stirring rate and amount and injection rate of the bridging liquid).

4.3. Effect of Feeding Position on Agglomerate Size (Series $E_{2}$ ). In the previous section, a correlation between $d_{43}$ of spherical agglomerates and the size of binder liquid droplets $d_{\text {drop }}$ was shown. Furthermore, to underline this correlation, a simple model (see Section 2.1) was employed for a theoretical estimation of the sizes of the liquid binder, $d_{\text {drop }}$. It has been shown that the present model can be used to estimate the size of bridging liquid droplets for different capillary sizes and provide a rough estimation of mean agglomerate size if other operating parameters (i.e., stirring rate, the amount and the injection rate of bridging liquid, etc.) remain unchanged. In this section, the same model is used to predict the effect of change of the velocity of the continuous phase. The model results are later compared with the experimental results (Series $\left.E_{2}\right)$.

In this work, the feed position $(z)$ was chosen as an operating parameter since the velocity of the continuous phase around the tip of a capillary varies for different positions (see Table 4). At the same time, other operating parameters were kept constant: capillary size $\left(d_{\text {in }}=320 \mu \mathrm{m}\right)$, flow orientation (co-flow), as well as the stirring rate (450 rpm) (see Table 3 ). As in the other experiments reported in this work, also in this case the stirring time, initial size, and amount of primary crystals as well as the amount and the injection rate of the liquid binder were kept constant. Therefore, the potential difference in agglomerate size can only be attributed to the difference in the droplet sizes.

In Figure 11, the estimated sizes of droplets $\left(d_{\text {drop }}\right)$ produced at different feed locations $(z)$ are displayed with star markers.

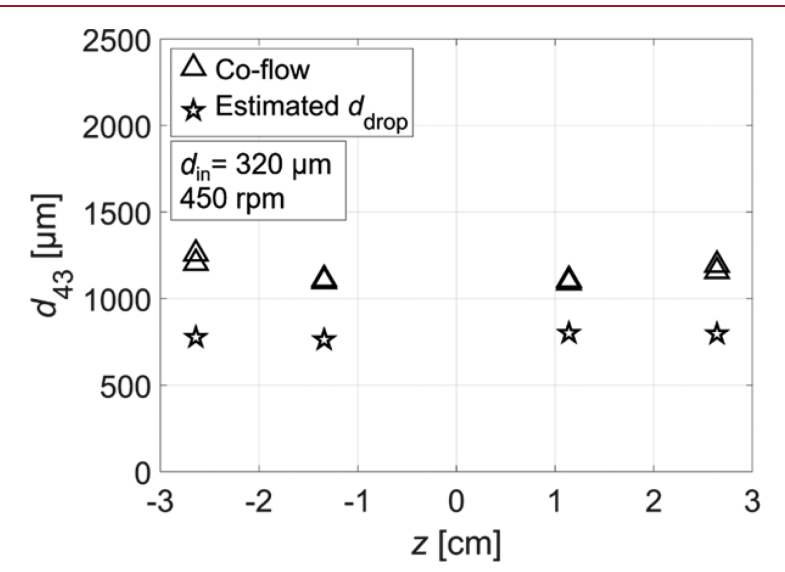

Figure 11. Effect of feed position $(z)$ on the volume-averaged size $\left(d_{43}\right)$ of spherical agglomerates (triangles) and on $d_{\text {drop }}$ estimated from eq 8 (stars) for experiments $E_{2}: d_{\text {in }}=320 \mu \mathrm{m}$, co-flow, stirring rate $=$ $450 \mathrm{rpm}$.

As shown in the figure, $d_{\text {drop }}$ values are similar for all four of the investigated locations, thus indicating that the corresponding change in the velocities of the continuous phase should have a negligible effect on $d_{\text {drop }}$ and hence on the final size of agglomerates, $d_{43}$, within the investigated conditions.

The PSDs of the final agglomerates obtained at different feeding locations are shown in Figure 12. The experimental results show very little variability between the different feeding locations. The experimental results of series $E_{2}$ are also shown in Figure 11 in terms of $d_{43}$.

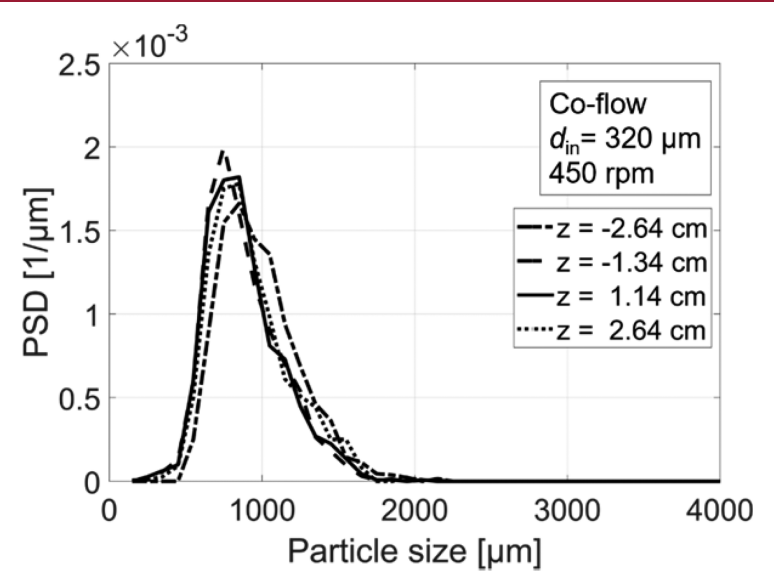

Figure 12. Effect of feeding position $(z)$ on the PSD of spherical agglomerates for experiments $E_{2}: d_{\text {in }}=320 \mu \mathrm{m}$, co-flow, stirring rate $=$ $450 \mathrm{rpm}$. All experiments were performed twice, yielding very similar PSDs. For the sake of clarity, only one of the two experimental results is reported for each experimental condition. 
4.4. Effect of Stirring Rate on the Size of Bridging Liquid Droplets and Agglomerates (Series $E_{3}$ ). As mentioned previously (see Section 1), numerous studies examined the effect of the stirring rate on the spherical agglomeration process. A number of papers have reported that an increase in the stirring rate results in an increase in the agglomerate sizes for small stirring rates and a size decrease for higher stirring rates. ${ }^{11,25}$ This effect could be explained by a change in the shear rate resulting in either a change in the dispersion of the bridging liquid, ${ }^{26}$ or in a change of the agglomeration kinetics affecting both the probability of collisions between particles as well as their contact time. ${ }^{27}$ In this section, we have used the model in a predictive manner to see if the behavior of the system where the stirring rate is varied can be captured by taking into account only the change in the velocity of the continuous phase-neglecting completely the aforementioned effect of the shear rate. The experiments were performed at three different stirring rates $(250,350$, and $450 \mathrm{rpm})$ while keeping other process parameters constant: capillary size $\left(d_{\text {in }}=320 \mu \mathrm{m}\right)$, flow orientation (co-flow), as well as the position in the reactor $(z=-2.64 \mathrm{~cm}$ ) (see Table 3 ). As in the other experiments in this work (see Sections 4.1 and 4.3), in this case the stirring time, initial size, and amount of primary crystals as well as the amount and the injection rate of the liquid binder were kept constant.

In Figure 13, the dependence of the estimated size of droplets $\left(d_{\text {drop }}\right)$ on the stirring rate is shown. As expected,

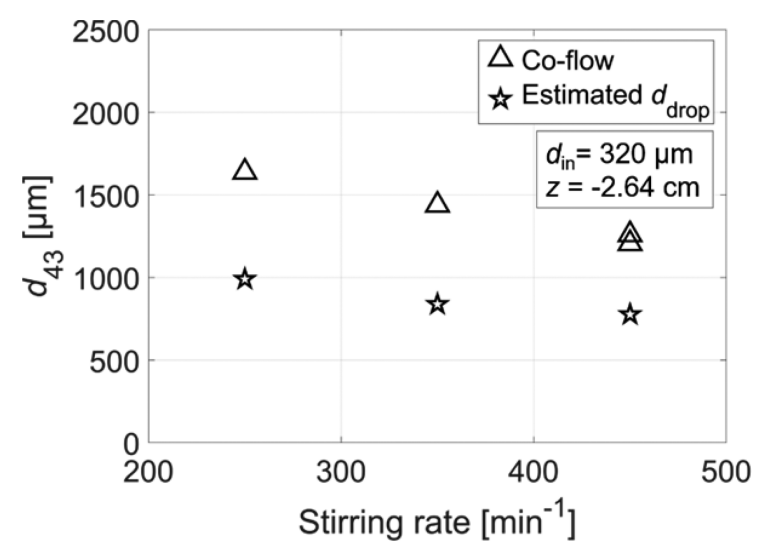

Figure 13. Effect of stirring rate on the volume-averaged size $\left(d_{43}\right)$ of spherical agglomerates (triangles) and on $d_{\text {drop }}$ estimated from eq 8 (stars) for experiments $E_{3}: d_{\text {in }}=320 \mu \mathrm{m}$, co-flow, $z=-2.64 \mathrm{~cm}$.

when increasing the stirring rate from 250 to $450 \mathrm{rpm}$ and thus increasing the velocity of the continuous phase (see Table 4), the $d_{\text {drop }}$ decreased, thus suggesting that the size of agglomerates should also decrease. The corresponding volume-averaged size $\left(d_{43}\right)$ of spherical agglomerates is also shown in Figure 13. As can be immediately seen, when the stirring rate is increased from 250 to $450 \mathrm{rpm}$, the $d_{43}$ indeed decreased from 1640 to $1230 \mu \mathrm{m}$. But, even though it seems that in general the behavior of the system can be captured correctly with the simple model also in this case, one should not forget that we have so far looked only at the effect of the first step of spherical agglomeration, namely, the wetting step, while neglecting the remaining two steps, which depend on the change in the energy dissipation rate as well (see Figure 10). When increasing the stirring rate, more particles are colliding, thus resulting in larger agglomerates. At the same time, the contact time is shortened and the compaction of particles due to collisions is enhanced-both resulting in smaller agglomerates. Since with the current experiments the contributions of those opposing effects on the final size of the spherical agglomerates cannot be quantified, the application of the simple proposed here model to qualitatively predict the final size of agglomerates in all cases should be done with caution.

\section{CONCLUSIONS}

In this work, the effect of the initial droplet size of bridging liquid on the final size of spherical agglomerates in a spherical agglomeration process has been experimentally investigated. By using a microfluidic device, it was possible to generate large quantities of binder droplets at a desired size, allowing for the decoupling of the characteristics of the initial binder dispersion from other critical operating parameters.

First, spherical agglomerates of benzoic acid of varying sizes using toluene as the bridging liquid were successfully obtained with a very good reproducibility. Second, it has been shown that by controlling the size of droplets of the bridging liquid, tuning the size of agglomerates was possible in a wide range of sizes. The effect of different process parameters on the size of droplets, mainly the size of capillary, flow orientation around the tip of the capillary, feeding position in the reactor, and stirring rate have been investigated.

Finally, a simple model has been used to estimate the size of bridging liquid droplets. The presented model adequately captures the behavior of the system and gives a qualitative agreement with the experimental results, thus allowing for a more precise design of particles using a spherical agglomeration process.

\section{ASSOCIATED CONTENT}

\section{Supporting Information}

The Supporting Information is available free of charge on the ACS Publications website at DOI: 10.1021/acs.cgd.8b01134.

High-speed camera video of the droplet formation process (AVI)

\section{AUTHOR INFORMATION}

\section{Corresponding Author}

*E-mail: marco.mazzotti@ipe.mavt.ethz.ch. Phone: +41 44 63224 56. Fax: +416321141.

ORCID $\odot$

Marco Mazzotti: 0000-0002-4948-6705

Notes

The authors declare no competing financial interest.

\section{ACKNOWLEDGMENTS}

This project has received partial funding from the European Research Council (ERC) under the European Union's Horizon 2020 Research and Innovation Programme under Grant Agreement No 2-73959-18. The authors thank Yan Wang, Tianjin University, for performing the initial experiments, and Ashwin Kumar, ETH Zurich, for fruitful discussions.

\section{NOMENCLATURE}

$A_{\text {ref }}$ reference area $\left[\mathrm{m}^{2}\right]$

$C_{\mathrm{D}}$ drag coefficient $[-]$

$d_{\text {drop }}$ droplet diameter $[\mathrm{m}]$ 
$d_{\text {in }} \quad$ inner capillary diameter $[\mathrm{m}]$

$d_{\text {out }}$ outer capillary diameter $[\mathrm{m}]$

$d_{43}$ particle size $[\mathrm{m}]$

$F$ force $[\mathrm{N}$ ]

$q_{\mathrm{d}} \quad$ volume flow rate $\left[\mathrm{m}^{3} \mathrm{~s}^{-1}\right]$

Re Reynolds number [-]

$u_{\mathrm{c}}$ continuous phase velocity $\left[\mathrm{m} \mathrm{s}^{-1}\right]$

$\bar{u}_{\mathrm{c}}$ average continuous phase velocity $\left[\mathrm{m} \mathrm{s}^{-1}\right]$

$u_{\mathrm{d}} \quad$ dispersed phase velocity $\left[\mathrm{m} \mathrm{s}^{-1}\right]$

$u_{\text {drop }}$ droplet velocity $\left[\mathrm{m} \mathrm{s}^{-1}\right]$

$u_{\text {rel }}$ relative velocity $\left[\mathrm{m} \mathrm{s}^{-1}\right]$

$\gamma \quad$ interfacial tension $\left[\mathrm{N} \mathrm{m}^{-1}\right]$

$\mu \quad$ dynamic viscosity $\left[\mathrm{N} \mathrm{s} \mathrm{m}^{-2}\right]$

\section{REFERENCES}

(1) Peña, R.; Nagy, Z. K. Process Intensification through Continuous Spherical Crystallization Using a Two-Stage Mixed Suspension Mixed Product Removal (MSMPR) System. Cryst. Growth Des. 2015, 15, 4225-4236.

(2) Kawashima, Y.; Cui, F.; Takeuchi, H.; Niwa, T.; Hino, T.; Kiuchi, K. Improvements in Flowability and Compressibility of Pharmaceutical Crystals for Direct Tabletting by Spherical Crystallization with a Two-Solvent System. Powder Technol. 1994, 78, 151157.

(3) Kawashima, Y.; Okumura, M.; Takenaka, H. The Effects of Temperature on the Spherical Crystallization of Salicylic Acid. Powder Technol. 1984, 39, 41-47.

(4) Thati, J.; Rasmuson, A. C. Particle Engineering of Benzoic Acid by Spherical Agglomeration. Eur. J. Pharm. Sci. 2012, 45, 657-667.

(5) Kawashima, Y.; Kurachi, Y.; Takenaka, H. Preparation of Spherical Wax Matrices of Sulfamethoxazole by Wet Spherical Agglomeration Technique Using a CMSMPR Agglomerator. Powder Technol. 1982, 32, 155-161.

(6) Blandin, A. F.; Mangin, D.; Rivoire, A.; Klein, J. P.; Bossoutrot, J. M. Agglomeration in Suspension of Salicylic Acid Fine Particles: Influence of Some Process Parameters on Kinetics and Agglomerate Final Size. Powder Technol. 2003, 130, 316-323.

(7) Morishima, K.; Kawashima, Y.; Kawashima, Y.; Takeuchi, H.; Niwa, T.; Hino, T. Micromeritic Characteristics and Agglomeration Mechanisms in the Spherical Crystallization of Bucillamine by the Spherical Agglomeration and the Emulsion Solvent Diffusion Methods. Powder Technol. 1993, 76, 57-64.

(8) Zhang, H.; Chen, Y.; Wang, J.; Gong, J. Investigation on the Spherical Crystallization Process of Cefotaxime Sodium. Ind. Eng. Chem. Res. 2010, 49, 1402-1411.

(9) Kawashima, Y.; Furukawa, K.; Takenaka, H. The Physicochemical Parameters Determining the Size of Agglomerate Prepared by the Wet Spherical Agglomeration Technique. Powder Technol. 1981, 30, 211-216.

(10) Thati, J.; Rasmuson, A. C. On the Mechanisms of Formation of Spherical Agglomerates. Eur. J. Pharm. Sci. 2011, 42, 365-379.

(11) Katta, J.; Rasmuson, A. C. Spherical Crystallization of Benzoic Acid. Int. J. Pharm. 2008, 348, 61-69.

(12) Zhu, P.; Wang, L. Passive and Active Droplet Generation with Microfluidics: A Review. Lab Chip 2017, 17, 34-75.

(13) Cramer, C.; Fischer, P.; Windhab, E. J. Drop Formation in a Co-Flowing Ambient Fluid. Chem. Eng. Sci. 2004, 59, 3045-3058.

(14) Scheele, G. F.; Meister, B. J. Drop Formation at Low Velocities in Liquid-Liquid Systems: Part I. Prediction of Drop Volume. AIChE J. 1968, 14, 9-15.

(15) Chuang, S. C.; Goldschmidt, V. W. Bubble Formation Due to a Submerged Capillary Tube in Quiescent and Coflowing Streams. J. Basic Eng. 1970, 92, 705.

(16) Abraham, F. F. Functional Dependence of Drag Coefficient of a Sphere on Reynolds Number. Phys. Fluids 1970, 13, 2194.
(17) Umbanhowar, P. B.; Prasad, V.; Weitz, D. A. Monodisperse Emulsion Generation via Drop Break Off in a Coflowing Stream. Langmuir 2000, 16, 347-351.

(18) Peng, S. J.; Williams, R. a. Part I: Droplet Formation from a Single Pore. Trans IChemE 1998, 76, 894-901.

(19) Zhang, X. Dynamics of Growth and Breakup of Viscous Pendant Drops into Air. J. Colloid Interface Sci. 1999, 212, 107-122.

(20) Rayleigh, L. On the Instability of Jets. Proc. London Math. Soc. 1878, s1-10, 4-13.

(21) González, B.; Calvar, N.; Gómez, E.; Domínguez, Á. Density, Dynamic Viscosity, and Derived Properties of Binary Mixtures of Methanol or Ethanol with Water, Ethyl Acetate, and Methyl Acetate at $\mathrm{T}=(293.15,298.15$, and 303.15) K. J. Chem. Thermodyn. 2007, 39, $1578-1588$

(22) Sada, E.; Kito, S.; Yamashita, M. Interfacial Tensions of TwoPhase Ternary Systems. J. Chem. Eng. Data 1975, 20, 376-377.

(23) Subero-Couroyer, C.; Mangin, D.; Rivoire, A.; Blandin, A. F.; Klein, J. P. P. Agglomeration in Suspension of Salicylic Acid Fine Particles: Analysis of the Wetting Period and Effect of the Binder Injection Mode on the Final Agglomerate Size. Powder Technol. 2006, 161, 98-109.

(24) Peña, R.; Burcham, C. L.; Jarmer, D. J.; Ramkrishna, D.; Nagy, Z. K. Modeling and Optimization of Spherical Agglomeration in Suspension through a Coupled Population Balance Model. Chem. Eng. Sci. 2017, 167, 66-77.

(25) Paradkar, A. R.; Pawar, A. P.; Chordiya, J. K.; Patil, V. B.; Ketkar, A. R. Spherical Crystallization of Celecoxib. Drug Dev. Ind. Pharm. 2002, 28, 1213-1220.

(26) Blandin, A. F.; Mangin, D.; Subero-Couroyer, C.; Rivoire, A.; Klein, J. P. P.; Bossoutrot, J. M. Modelling of Agglomeration in Suspension: Application to Salicylic Acid Microparticles. Powder Technol. 2005, 156, 19-33.

(27) Amaro-González, D.; Biscans, B. Spherical Agglomeration during Crystallization of an Active Pharmaceutical Ingredient. Powder Technol. 2002, 128, 188-194. 